\title{
CARRIER SCREENING FOR SINGLE-GENE DISORDERS - A BRIEF REVIEW
}

\author{
Katya S. Kovacheva, \\ Slavena E. Nikolova, \\ Zornitsa B. Kamburova
}

Department of Medical Genetics, Medical University - Pleven

\section{Corresponding Author:}

Slavena E. Nikolova

Department of Medical Genetics, Medical University - Pleven

1, Saint Kliment Ohridski Str.

Pleven, 5800

e-mail: sl_nikolova@abv.bg

Received: September 30, 2021

Revision received: October 10, 2021

Accepted: November 25, 2021

\section{Summary}

Carrier screening (CS) is an approach to pre-reproductive identification of couples at risk of having offspring with a single-gene disorder (SGD). The goal of CS is to facilitate reproductive autonomy and informed decision-making of the revealed risk couples. Initially, CS was introduced in the 1970s among the Ashkenazi Jewish population in the USA with a high incidence of Tay-Sachs disease. Until recently, CS had a limited application, mainly among individuals of a particular ethnicity or family history of SGD, and a limited number of conditions are included. Implementing the revolutionary technology of next-generation sequencing revealed a much more comprehensive range of possibilities for CS. The expanded alternative variant introduced a new concept that allows screening for hundreds to thousands of SGDs without pre-selection of individuals based on their ancestry or family history. Furthermore, the number of detected pathogenic variants is much higher compared to the traditional CS. Currently, there is insufficient experience with the application of expanded CS, and the lack of uniform guidelines is a major problem when it comes to massive implementation. Nevertheless, the significant potential of the expanded CS has given rise to a growing interest in it and provoked in-depth discussions about the opportunities of widespread application in practice.

Keywords: carrier screening, recessive disorders, ethnicbased, universal, expanded

\section{Introduction}

Genetic disorders are gradually gaining more attention during the last decades, primarily due to the remarkable advances in molecular technologies allowing a better understanding of genetic mechanisms and the achievement of more adequate diagnoses. Single-gene disorders comprise approximately 39\% of all rare conditions $[1,2]$. It is estimated that they collectively account for $10 \%$ of infant mortality and between $10 \%$ and $34 \%$ of pediatric hospitalizations [3]. Recessive single-gene disorders (autosomal and $\mathrm{X}$-linked) constitute a rather significant proportion of all mendelian diseases. Even though their true prevalence is still unknown, it is considered that there are more than 1700 recessively inherited conditions 
[4]. In the group of autosomal recessive (AR) conditions, the predominant transmission type is via asymptomatic heterozygous parents unaware of their carrier status. The parents have a recurrence risk (constant for each pregnancy) of $25 \%$ for a sick child. In contrast, there is a constant risk of $50 \%$ for each son of an asymptomatic heterozygous mother in cases of $\mathrm{X}$-linked recessive disorders.

A well-noted risk factor for congenital malformations and AR conditions is consanguinity. More than 1.2 billion people of the current global population practice consanguineous marriages, a union between first cousins being the most common. Such mating couples share one-eighth of their genes inherited from a common ancestor, which results in their progeny being homozygous at $1 / 16^{\text {th }}$ of all loci [5]. This homozygosity explains the increased risk for affected offspring of consanguineous parents. Even though non-consanguineous marriages are less exposed to the risk regarding recessive conditions, they should not be overlooked. Every individual is likely to be a carrier for at least one such mutation, approximately 2.8 recessive mutations per individual [6]. The estimated risk of every heterozygous couple being affected with an AR condition homozygous offspring is $2.5 \%$. In a consanguineous couple, for second cousins and first cousins, the risk is $6.9 \%$ and $22.6 \%$, respectively [7]. Cumulatively recessive conditions affect at least 30 in every 10000 children, which means that approximately 1-2 in 100 couples are at risk of having an affected offspring [8]. There is a well-defined understanding of the health burden of AR conditions and single-gene disorders in general. These disorders significantly impact multiple aspects, including the patient's health status, quality of life and life expectancy, and a family's financial and psychological well-being. It is noteworthy that these disorders require multiple hospitalizations leading to substantially higher healthcare paid claims than any other group of patients [9]. Therefore, due to all listed evidence, the best way to manage recessive conditions would be to put efforts into prospective testing and identifying asymptomatic carriers. Carrier screening is a well-established clinical initiative that has been implemented for decades. Currently, it is a significant point of discussion amongst different medical fields worldwide, mainly because of significant changes in laboratory capacities and the introduction of high-throughput sequencing.

This survey aims to present a brief review of the history, basics, and current perspectives (potential benefits and challenges) of carrier screening programs and their application worldwide.

\section{Methods}

A comprehensive literature search was conducted using many terms and topic-related keywords: carrier screening, recessive disorder, ethnic-based, universal, expanded. The search was predominantly carried out in two database sites: ResearchGate and PubMed. Only English language sources were used.

\section{History and basics of targeted carrier screening}

According to the definition of the American College of Obstetricians and Gynecologists (ACOG), carrier screening (CS) is genetic testing performed on an individual who does not have any overt phenotype for a genetic disorder, yet may have one variant allele within a gene or genes associated with a diagnosis [10]. The European Society of Human Genetics (EJHG) defines $\mathrm{CS}$ as a medical investigation aimed to detect whether or not a carrier status for a recessive disorder is present in a couple or a person who does not have an apriori increased risk of being a carrier based on their or their parents' personal or family disease history [8].

In the past, the original goal of CS was to reduce the incidence of conditions with severe manifestation. In 1982, Ian Porter stated that the main purpose of CS was prevention through genetic counseling for reproductive options. Not too long after that, the statement was denounced due to ethical concerns. Using the word prevention as suggesting that the preferable outcome is the reduction of affected births, incidence does not match the reproductive option initiative. On the other hand, the prevention strategy raises issues regarding people with such conditions and disabilities and potential discrimination against them. That led to dismissing "prevention" when addressing the 
primary objective of CS. The current commonly accepted and agreed upon purpose of CS is facilitating informed reproductive autonomy and decision-making of prospective parents, rather than emphasizing early diagnosis and treatment. However, an expected outcome would be the incidence reduction in couples who choose to pursue prevention [11]. A couple's autonomy is enhanced when the partners are informed about the risk of having an affected child and all the alternatives they can choose when high risk is confirmed.

Traditionally, CS was performed within specific ethnic groups with a high prevalence of certain AR conditions. Following this initial manner, the very first implementation of CS in 1971 involved people of Ashkenazi Jewish (AJ) descent in the USA. In this group, there was a high carrier frequency of Tay-Sachs disease (TSD) -1 in 30 compared to 1 in 300 in the general population and 100 times higher prevalence of affected individuals. TSD is a lysosomal storage disorder caused by a deficiency of $\alpha$ subunit of the $\beta$ hexosaminidase enzyme, resulting in GM2 ganglioside neuronal accumulation. It is characterized by progressive neurodegeneration, leading to early childhood death in individuals with the infantile, and a delayed clinical course in individuals with juvenile or adult-onset of the disease 12]. Population-based CS was performed by measuring Hex A enzyme activity in serum or leukocytes in a blood sample. The technique is inexpensive and highly sensitive, with a detection rate of $98 \%$. These early screening programs were shown to be quite successful as they managed to reduce the prevalence of TSD by more than $90 \%$ in the United States and Canada - from approximately 60 affected born per year to 3-5. Even though enzyme testing has proven effective, some limitations have been pointed out, including false-positive and intermediate results. In the 1990s, direct DNA analysis of the HEXA gene was also implemented in addition to enzyme testing, offering a highly specific and even more reliable approach. According to National Tay-Sachs and allied disease association (NTSAD), every positive result requires additional confirmation molecular testing to define the underlying pathogenic variant. At first, three common gene variants were tested as they are prevalent in the AJ community, accounting for up to $98 \%$ of the pathogenic variants. Ever since, the number of target variant panels has grown, as well as the number of conditions (up to 38 disorders) for which AJ individuals have been screened [13]. However, there is one considerable limitation: a limited set of pre-determined variants is detected. Moreover, this approach may prove to be less efficient when it is used to screen people of a descent different from $\mathrm{AJ}$ descent.

Beta-thalassemia is another very wellknown AR condition for which large-scale CS is performed. The condition is characterized by decreased production of beta-globin chain, resulting in an unbalanced $\alpha / \beta$ globin chain ratio. Along with severe anemia, clinical manifestation involves multiple organs and systems. Persons with beta-thalassemia major live an average of 17 years and usually die by 30 years of age [14]. The treatment requires regular $\mathrm{RBC}$ transfusions, iron chelation, and management of secondary complications of iron overload. Bone marrow transplantation remains the most effective one. Beta-thalassemia is prevalent in Mediterranean countries, the Middle East, Central Asia, India, Southern China, the Far East, and countries along the north coast of Africa and South America. The highest carrier frequency is reported in Cyprus (14\%), Sardinia (10.3\%), and Southeast Asia. The annual incidence is 70000 , and the estimated number of carriers across the world is $80-90$ million individuals, comprising $1.5 \%$ of the global population. The massive number of carriers and presence of target regions with a higher frequency of affected individuals could be explained with a natural selection against Plasmodium falciparum, the causative agent of malaria [15].

Voluntary premarital carrier screening for beta-thalassemia began in 1973 in Cyprus and two years later, in Sardinia. Both places have sustainable programs for thalassemia awareness and control [16]. In Cyprus, the carrier frequency was $1: 7$, the expected frequency of carrier couples was $1: 49$, the prevalence of affected individuals at that time was 1 in 1000 [17]. The primary diagnosis of heterozygous carriers is based on analyzing two hematological parameters (MCV and $\mathrm{MCH}$ ) and subsequent $\mathrm{HbA} 2$ measurement with electrophoresis or high-performance liquid chromatography [18]. The implementation of the screening program for beta-thalassemia was successful in identifying the heterozygous 
carriers. Between 1974 and 1979, the annual number of born with the condition individuals dropped from 51 to 8 . In the early 1980 s, the CS for beta-thalassemia in Cyprus acquired a quasimandatory status - the premarital screening was mandated by the Cypriot Orthodox Church. As a result of implementing these changes, between 1991 and 2001, only five affected births were documented, and no affected births occurred between 2002 and 2007 [19]. The estimated frequency of at-risk couples in Sardinia was 1 in 60 [20]. After implementing the screening program, the incidence dropped from 1:250 to $1: 4000$, i.e., by approximately $94 \%$. In other countries with CS programs for beta-thalassemia, such as Taiwan, China, France, United Kingdom, and Canada, there was a significant reduction in the incidence of affected births [19]. Like TSD, the carrier diagnosis for beta-thalassemia was based primarily on hematologic analysis and DNA analysis to confirm the pathogenic variant [21].

Cystic fibrosis is estimated to be the most common AR condition in populations of European descent. The average birth prevalence is 1 in 2500-3500, and the carrier frequency is 1 in 25-30 [22]. Mutations cause the disorder in the cystic fibrosis transmembrane conductance regulator (CFTR) gene, leading to abnormal expression of its product - the CFTR protein. It is an ion channel in the epithelial surface of the airways, pancreatic ducts, intestines, bile ducts, and sweat glands. The usual clinical manifestation includes chronic lung disease, exocrine pancreas insufficiency, and elevated sodium chloride levels in the sweat. The average life expectancy is 37.4 years [23]. After the identification of the CFTR gene in 1989, CS was implemented.

After the National Institute of Health (NIH) conference in 1997, a consensus stated that CS should be recommended to individuals with family history, partners of individuals with $\mathrm{CF}$, and prospective parents [24]. Outside of the USA, countries like UK, France, and Canada still refrain from population-based screening and recommend it only to individuals with family history. The ACOG recommendations shifted from all women of Eastern European and Northern European descent to all women to be screened regardless of their ancestry. More than 2000 mutations have been listed, delF508 being the most common and accounting for $70 \%$ of all CFTR alleles in affected individuals with Northern European ancestry [25]. Currently, the American College of Medical Genetics (ACMG) and ACOG recommend a 23-mutation panel testing for all mutations whose frequency is no lower than $0.1 \%$ in the general population of the USA. Universal CF CS has been predominantly offered to pregnant women during their routine clinic visits. The reported uptake of the women is $46-99 \%$. Approximately $80-100 \%$ of identified at-risk couples underwent a prenatal diagnosis, and almost all pregnancies with confirmed CF were selectively terminated according to the parents' decision. However, the CS for CF has demonstrated a modest reduction in the incidence of CF [8].

\section{Advantages and limitations of targeted screening}

The examples above illustrate the traditional targeted CS program. Along with including individuals of specific racial or ethnic origin, the program is also conducted if there is a family history of AR condition. It uses laboratory methods or genotyping of pre-selected gene variants that are common in a particular population. Targeted carriers screening tends to be less expensive due to fewer individuals screened (CA Rowe). It proves effective as the primary goal, i.e., reducing the incidence of affected births, is accomplished and has indisputable advantages. However, the target CS currently tends to be outdated because there is a gradually increasing number of reported limitations:

1) One major issue would be difficult to assign an individual to a single ethnicity due to everincreasing numbers of inter-ethnic marriages and individuals of mixed racial ancestry.

2) Lack of knowledge about ancestry due to unknown parents or all four grandparents, or incomplete disclosure of family history or carrier status. For example, $40 \%$ of US citizens aged 18-64 are unaware of the descent of their four grandparents.

3) Emphasizing a given race or ethnic group can lead to stigmatization and racial or ethnic bias.

4) Conventional genotyping targets limited 
pre-determined disease-causing variants, which are common in the populations screened. Actually, most of the conditions tested are caused by multiple pathogenic alleles, sometimes up to thousands, and such panels do not cover all potential variants. When used in the settings of a pan-ethnic population, these tests are much less informative. This disadvantage has been demonstrated with the AJ panels used outside the AJ population, where target screening tends to have a significantly reduced sensitivity.

5) Genetic conditions can be recorded at significantly higher rates in some populations, but they also have a lower frequency amongst individuals of different ethnic origins. Focusing solely on several ethnic groups and neglecting individuals outside the populations in question and those without a family history can potentially miss many at-risk couples. This statement was supported by a 2013 research based on screening 23,453 individuals of different ethnic origins. About $24 \%$ of all tested individuals were identified as carriers for at least one of 96 severe recessive conditions, and $5.2 \%$ were carriers for multiple conditions.

6) Lack of unification in the different guidelines and not enough statements concerning other than ethnic-based CS. For example, 75\% of the carriers found in a research of Gabriel A. Lazarin, MS, from 2013 would not be identified if ACOG or ACMG guidelines were used.

7) Insufficient information regarding the testing because it targets a limited number of individuals [27, 28, 29, 30].

\section{Universal carrier screening}

A different approach for CS set to deal with some of the limitations of the target-group approach is the universal or pan-ethnic CS. It offers to test all individuals regardless of racial and ethnic origin or family history. Currently, disorders that are recommended as suitable for universal CS are cystic fibrosis, spinal muscular atrophy (ACOG, ACMG) [10, 31], and hemoglobinopathies (ACOG) [10]. All of these conditions are highly prevalent in affected births and have high carrier frequency worldwide. The strategy implies developing a single, sensitive, and specific panel for the detection of pathogenic variants. The panel is supposed to combine the accuracy and utility of several single-gene assays. It is important to note that the universal CS is intended to reduce disease risk rather than eliminate it completely. According to Balaji S. Srinivasan et al., the main advantages of this approach are:

1. easier to implement in clinical practice;

2. reduction of racial and ethnic discrimination and higher equity;

3. the potential to identify a higher number of carriers without targeting a particular population;

4. testing both larger and smaller populations with a unified panel, thus minimizing stigmatization of a genetic condition as an ethnic-specific problem [32].

The main disadvantages of universal CS are higher costs, the necessity of introducing and training many healthcare professionals, and the unification of laboratory methods everywhere the screening is performed [26].

\section{Expanded carrier screening}

Genomic technologies underwent considerable development after the human genome project, leading to next-generation sequencing (NGS). The high-throughput sequencing demonstrates several significant advantages compared to traditional genotyping, which has revolutionized genetic testing. These advantages have served as a reason for its quick implementation.

Massive parallel sequencing of multiple targeted genomic fragments in multiple samples became possible. This is especially suitable because the number of Mendelian disorders is constantly increasing. Besides, individually discussing the samples is not cost- and timeeffective. Another essential advantage is using this approach for conditions with genetic heterogeneity.

The high-throughput sequencing improves utility as it is not designed only to identify preselected common known mutations. Within one diagnostic assay, several thousand variants are tested. Along with pathogenic variants, it can detect variants of unknown significance. Both sensitivity and specificity are greatly improved.

Another advantage relates to the significantly reduced prices and considerable costeffectiveness [33].

Advances in bioinformatics allow for its multiple applications in scientific and medical fields [34]. CS can also greatly benefit, as 
the expanded universal CS got introduced in 2009 [35]. NGS acts as a catalyst, enabling the transition from a targeted approach of detecting carriers of limited conditions with a particular racial or ethnic background to testing hundreds to thousands of different genes in multiple individuals regardless of their ancestry or family history [36]. There is a well-noted similarity between expanded and universal CS - escaping the emphasis of testing a specific population and availability for much greater numbers of individuals. Expanded carrier screening (ECS) also solves stigmatization and racial or ethnic bias problems, thus providing equity [8]. Another important difference is that ECS offers screening for a much greater number of conditions with a methodology of better analytical accuracy than traditional genotyping. This can dramatically increase the number of identified at-risk couples and, respectively, provide valuable information to individuals who can benefit from it. Several studies have demonstrated that NGS outperforms traditional genotyping in both expanded and targeted CS.

ECS is gradually gaining popularity across the world as more data of pilot studies is getting published. The growing interest can be explained by the increasing access to NGS technologies and a better understanding of the remarkable opportunities it holds. Besides, there is an undeniable necessity to address the current pending questions and challenges related to ESC implementation.

The most important question that has been raised since the implementation of ECS is what conditions have to be included on the screening panel. Even though guidelines from professional organizations regarding panel design exist, there are currently no unified criteria to be followed. This was demonstrated by a study comparing 16 ECS providers. The included conditions varied from 41 to 1792 , with an overlap of only three conditions [37]. According to ACOG, when designing an ECS panel, the conditions included should have a well-defined phenotype, a detrimental effect on life quality, cause cognitive or physical impairment, require surgical or medical intervention, or have an onset early in life. One overt difficulty is to define detrimental effect and severe manifestation. Three significantly broad studies conducted in
2011 and 2016 designed panels with conditions defined as ,severe, ', great,' and ,profound, ' and showed drastic differences. Bell et al. included a total number of 448 disorders, and Haque et al. picked 93, while the study of Plantinga et al. included only 50 . Only 30 of all conditions were present in all three panels [26]. A study by Lazarin et al. in 2014 proposed a set of criteria to be used when classifying a condition as profound, severe, and moderate [38].

There is no unanimous opinion regarding including conditions with a mild phenotype as it raises moral issues. So far, there is no clear answer to whether it is necessary to terminate a pregnancy or avoid conceiving a child with a disease that does not cause unbearable suffering but still alters the quality of life [11]. According to ACMG, conditions of mild clinical impact and those with variable expression and reduced penetrance should be optional rather than routine. Different statements are given regarding adult-onset conditions. According to ACOG and ESHG, these conditions should not be included. In contrast, ACMG states that whenever such conditions are included, a patient's consent is required.

Another supposed aspect for conditions selection on the panel is the carrier frequency and the prevalence of a condition, but it is vaguely addressed and discussed. So far, ACOG is the only official society that has a statement regarding this particular issue. According to ACOG, the admissible carrier frequency is 1 in 100 or greater across all ethnicities. ACMG's statement about carrier frequency is addressed regarding the estimation of residual risk. ESHG does not address carrier frequency or affected births incidence. Individual studies also tend to follow their own pre-selected frequencies. For example, the panel of Platinga et al. is designed to detect conditions with an incidence of 1 in 600 [39], whereas Bell et al. did not address the low incidence of conditions in their panel [40].

A study by Rotem Ben-Shachar et al. from 2019 states that ACOG's 1-in-100 carrier frequency criterion leaves room for interpretation - a condition with 1-in-100 or greater carrier rate in any ethnicity, a condition with 1-in-100 or greater carrier rate when ethnicities are weighed by their US census frequencies or a condition with 1-in-100 or greater carrier rate in all 
ethnicities. In these three settings, the use of this frequency has been shown to limit the detection of at-risk couples. There are rare conditions with significantly lower carrier frequency yet demonstrating a considerable detection rate due to a low number of pathogenic variants. Some conditions with high prevalence have a limited detection rate due to a higher number of pathogenic variants. Also, the carrier frequency is a suboptimal metric because an individual carrier result is insufficient to estimate the reproductive risk. The risk estimation requires both partners' carrier status. Another commonly discussed metric for panel design is the atrisk couple frequency - the square of a carrier frequency for AR conditions [37]. This type of frequency is inapplicable for particular singlegene conditions with an unusual pattern of inheritance. Because of the above-listed reasons, "modeled fetal disease risk" is a more suitable metric suggested in designing a panel for ECS purposes. It illustrates the disease probability in a conceptus of a randomly selected couple [41].

Even though ECS's main advantage is the potential to screen individuals for a considerably high number of conditions, it is suggested that there is a need for a specific limit. The limitations are not defined as to what the maximal admissible number is. Still, it is not advisable to include too many conditions, especially those with mild or variable phenotype or reduced penetrance, or genes involved with conditions with multifactorial etiology [42]. This can potentially lead to an unjustified rise in the cost of the testing and possible failure to estimate residual risks whenever there is no well-defined genotype-phenotype correlation. In addition, ECS can worsen patients' anxiety [43]. ACMG clearly states the necessity to stick to distinctly pre-defined criteria when designing a panel and dismiss the ,include as many disorders as possible' approach. Such an approach is allowable only in the settings of prospective pilot studies when determining which conditions and genes, respectively, should be selected for a panel to use within a defined population [44].

Another essential question to address is what the preferred timing for screening should be. There is widespread agreement on preconception screening as the optimal approach. It utilizes the best of all available options in terms of reproductive decision-making if the couple is atrisk. CS during pregnancy has fewer available options if it identifies the couple to be at-risk.

According to a report of Human Genetics Commission from 2011 on preconception genetic testing and screening, the primary reproductive options are as listed:

Before conception:

1. Undergoing in vitro fertilization with preimplantation genetic diagnosis of embryos and subsequent transfer of unaffected ones. This option virtually eliminates the risks of conceiving an offspring with the disease in question

2. Natural conception with subsequent prenatal diagnosis (chorion villus sampling or amniocentesis) to confirm or dismiss the diagnosis in the fetus.

3. Once the diagnosis is confirmed, the pregnancy can be terminated if the couple decides to do so. Even if the couple decides not to change the course of pregnancy, the information is still beneficial as instant measures can be pursued right after the delivery.

4. Natural conception without prenatal diagnosis and accepting the possibility of having an affected offspring.

5. Conception using donor sperm/egg.

6. Adoption.

7. Remain childless. During pregnancy:

1. Undergoing prenatal diagnosis (chorion villus sampling or amniocentesis according to the weeks of gestation) to confirm or dismiss the diagnosis in the fetus. Once the diagnosis is confirmed, the pregnancy can be terminated if the couple decides to do so. Even if the couple decides not to change the course of pregnancy, the information is still beneficial as instant measures are pursued right after the delivery.

2. Not pursuing a prenatal diagnosis and accepting the possibility of having an affected offspring [26].

The third aspect deserving attention is whether ECS should be performed on couples or individuals. Different options have been offered in this regard, each with its advantages and disadvantages. The two main types of CS are individual- and couple-oriented screening. 
The individual approach has been broadly implemented as it is acquired from the targeted CS [45]. Since the test is applied to an individual, he/she will always have the results disclosed to $\mathrm{him} / \mathrm{her}$. Subsequently, the information about the carrier status can be used for cascade screening, i.e., testing of first- and seconddegree relatives of an identified carrier, because relatives can share many common variants $[45$, 46]. For example, the siblings of a carrier have a $50 \%$ risk of being carriers, too [47]. However, it is essential to note that, nowadays, various studies agree that cascading screening is no longer an appropriate approach to consider [46, 48]. This is related mainly to the fact that every individual is a potential carrier [26]. Currently, both providers and researchers share the opinion that individual screening is not suitable in the settings of population-based ECS. Individual carrier status has reduced clinical utility because reproductive decision-making is based solely on the status of both partners. It does not contribute enough when estimating an offspring's risk.

Furthermore, individual disclosure of carrier status can potentially cause anxiety and perception of illness in detected carriers. Another documented disadvantage of the individual approach is more time-consuming counseling when reporting the results. All listed points make it reasonable to consider undertaking a couple's screening as more practical and adequate. It is generally assumed to be much better at utilizing the concept of ECS. This approach allows for both partners to be tested simultaneously. It can be very beneficial when performed during pregnancy because simultaneous detection of carrier status in both partners can imply prenatal diagnosis. It is still debated how the results should be disclosed - whether at-risk couples should obtain information beyond the carrier state for other individual pathogenic variants or whether couples that are not at-risk should obtain information regarding individual pathogenic variants [48]. Additional advantages include reduced anxiety and minimized followup counseling. Another approach that has been used is sequential screening, with females from couples being tested first and males tested afterward for selected genes based on the results of their partners. So far, the sequential screening is not favored due to well-noted disadvantages, such as increased time and anxiety (especially crucial during an ongoing pregnancy), disproportionately shared between the partners [46].

\section{Challenges (limitations) of ECS}

Even though ECS holds great potential in identifying multiple at-risk couples and enhancing their reproductive autonomy, it is worth noting that there are challenges that are yet to be resolved. The NGS technique used for ECS allows whole genes to be sequenced instead of selected sequence variants. It demonstrates certain advantages compared to conventional single-gene genotyping. The carrier detection rate is significantly high - 98-99\%. Nevertheless, it is crucial to point out that ECS cannot provide a $0 \%$ risk for the couples to have an affected child. For example, suppose a patient of Ashkenazy Jewish descent with a pre-test carrier risk of 1 in 25 undergoes cystic fibrosis screening with a detection rate of $94 \%$. In that case, their carrier risk after a negative screen is reduced to 1 in 380 [27]. In cases of negative CS results, there is always a residual risk (RR), although it is generally low. RR illustrates the likelihood of an individual with a negative result being an actual carrier. Some mutation variants of deletion/duplication type can be more difficult to detect (incomplete sensitivity). Whole-exomesequencing allows for identifying variants within the coding parts of the gene, whereas some alterations can occur within regulatory gene regions. Besides, ECS provides limited information as there are conditions that are not included in the panel.

$\mathrm{RR}$ of a particular condition is estimated by using the carrier frequency and detection rate of that condition in the following formula - carrier frequency x (1 - detection rate (DR)) [43]. Therefore, an accurate calculation of both mentioned metrics' values is needed. Carrier frequency is the proportion of individuals who carry a single heterozygous pathogenic variant for a recessive genetic condition in a given population. DR is the sum of disease allele frequencies for a given disease gene that could be detected by a specific molecular screening method. It has been proposed that to minimize the RR, two DR-related factors need to be taken into consideration: analytical detection rate and 
clinical detection rate. The first one illustrates the ability to detect pathogenic variants accurately.

In contrast, the latter one illustrates the ability to determine the clinical impact of a given variant, whether benign or pathogenic. The second factor needs a precise evaluation and update as more new variants are detected [49]. To obtain accurate data, ECS providers must constantly publish their own data, which, unfortunately, is not an established practice [50].

To design an adequate panel for ECS, RR is an essential criterion that is starting to be recognized. It still fails to be addressed appropriately and is yet to be improved. Inconclusive carrier results present another challenge of ECS that needs attention. Along with pathogenic/likely pathogenic and benign/likely benign variants, there is an additional classification of variants with unknown significance (VUS) [51]. Such results make it difficult for clinicians to interpret data of such sort and how to bring it to risk estimation, which would lead to confusion and anxiety in patients about their reproductive decisions [52]. To this day, there are no unified guidelines on how to deal with VUS [26].

Introducing the patients to the essence of ECS and revealing the results within counseling is yet another debatable issue. It is generally agreed that both pre-test- and post-test counseling is necessary. A significant component of pre-test counseling is obtaining informed consent from the couple to be tested. According to a joint statement of few professional organizations, the main points of this consent should include:

1. Voluntary nature of the test;

2. Confidentiality of the results;

3. Explanation of the clinical impact of the conditions for which the patients will be tested;

4. Information about the accurate risk estimation depending on proper paternity knowledge;

5. Information about the residual risk associated with negative results;

6. Explanation of the impact of being a carrier;

7. Explanation of possible identification of homozygous and/or heterozygous carrier states that can affect the individual's health status [53].

During post-test counseling, the test results and all reproductive options have to be fully revealed to the patients. Given the specificity and high standards of the test itself and the interpretation of results, either a medical geneticist or a genetic counselor must conduct the counseling. This is difficult to accomplish due to the small number of genetic specialists around the world. According to a study from 2018, there are approximately 7000 genetic counselors in 28 countries. About 4000 of them are in the United States alone (1 for every 82000 persons) [54]. Currently, the significant portion of medical specialists conducting genetic counseling comprises obstetricians, midwives, and general practitioners. All these healthcare providers are required to undergo appropriate education and training. It is a challenging task, considering the rapid growth of the medical genetics field. Furthermore, few medical specialists feel comfortable conducting counseling for the purpose of ECS, so they refrain from offering it to patients or else provide it when patients require it [55].

As previously mentioned, one of the main advantages of ECS is general testing of all individuals due to difficulties in assigning an individual to a single ethnicity. However, for specific populations, there is still a considerable homogeneity. In that sense, one single panel for everyone would not be suitable, and ECS providers should still consider the genetic characteristics of the population they serve [57].

Stephanie A. Kraft et al. [57] discussed additional challenges that should be worked on to implement the ECS fully. One of these challenges is the lack of interest in the general public that can be explained by the lack of awareness and knowledge about CS or the reluctance of people to cause stress to themselves. In general, health care systems are unlikely to recognize CS as important because they focus on serving individuals with an overall good health status rather than treating patients who already have an illness. It can also give rise to social disapproval and be possibly perceived as a form of eugenics, considering persons who already have the genetic conditions for which couples are screened. Another concern is about ECS being perceived as a mandatory act rather than a voluntary one. 


\section{Conclusion}

Recent decades focus on genetic disorders, including single-gene conditions, as one major cause of morbidity and mortality. Since these conditions are a significant burden and have a detrimental effect on the quality of life of affected individuals and their families, proper management would be prospective detection of carrier couples at high risk of having an affected offspring. The targeted CS strategies have been proven to be highly effective in reducing the incidence of recessive single-gene conditions. Currently, targeted CS seems to be less suitable as it has been shown to have some significant limitations. Recent advances in molecular technologies introduced high-throughput sequencing, which revolutionized the field of molecular testing and diagnosing. It allowed for implementing an alternative of CS in the form of ECS, the latter capable of managing some of the major disadvantages of targeted CS. Even though there are undeniable advantages such as low-cost effectiveness, simultaneous sequencing of multiple DNA fragments of multiple samples, and improved utility, there are pending challenges. However, there are pending challenges that are yet to be managed. A major point to be worked on suggests uniform guidelines, as the current lack of such causes considerable difficulties in optimal usage of ECS.

\section{Conflict of Interest}

The authors declare no conflict of interest.

\section{Acknowledgement}

A diagnostic genetic test will be performed on all family members in the frame of the project BG05M20P001-1.002-0010 „CENTER OF COMPETENCE IN PERSONALISED MEDICINE, 3D AND TELEMEDICINE, ROBOT - ASSISTED AND MINIMALLY INVASIVE SURGERY“, funded by the „Science and Education for Smart Growth“ Operational Program and the European Regional Development Fund, with Medical University Pleven as the leading organization.

\section{References}

1. Ferreira, CR. The burden of rare diseases. Am J Med Genet. 2019;179A:885-92.

2. Wakap SN, Lambert DM, Olry A, Rodwell C, Gueydan C, Lanneau V et al. Estimating cumulative point prevalence of rare diseases: analysis of the Orphanet database. Eur J Hum Genet. 2020;28(2):165-73.

3. Kumar P, Radhakrishnan J, Chowdhary MA, Giampietro PF. Prevalence and patterns of presentation of genetic disorders in a pediatric emergency department. Mayo Clin Proc. 2001;76(8):777-83.

4. Kirk EP, Barlow-Stewar K, Selvanathan A, Josephi-Taylor S, Worgan L, Rajagopalan S et al. Beyond the panel: preconception screening in consanguineous couples using the TruSight One "clinical exome". Genet Med. 2019;21(3):60812.

5. Fareeda M, Afzala M. Genetics of consanguinity and inbreeding in health and disease. Ann Hum Biol. 2017;44(2):99-107.

6. Bell CJ, Dinwiddie DL, Miller NA, Hateley SL, Ganusova EE, Mudge J et al. Carrier testing for severe childhood recessive diseases by nextgeneration sequencing. Sci Transl Med. 2011 Jan 12;3(65):65ra4.

7. Antonarakis, SE. Carrier screening for recessive disorders. Nat Rev Genet. 2019;20(9):549-61.

8. Henneman L, Borry P, Chokoshvili D, Cornel $\mathrm{CC}$, van El CG, Forzano F et al. Responsible implementation of expanded carrier screening. Eur J Hum Genet. 2016;24(6):e1 e12.

9. Miller KE, Hoyt R, Rust S, Doerschuk R, Huang Y, Lin SM. The financial impact of genetic diseases in a pediatric accountable care organization. Public Health Front. 2020;8(58).

10. ACOG Committee opinion No. 691 Summary: Carrier Screening for Genetic Conditions. Obstetrics \& Gynecology. 2017;129(3):597-9.

11. van der Hout S, Dondorp W, de Wert G. The aims of expanded universal carrier screening: Autonomy, prevention, and responsible parenthood. Bioethics. 2019;33(5):568-76.

12. Mehta N, Lazarin GA, Spiegel E, Berentsen K, Brennan K, Giordano J et al. Tay-Sachs Carrier Screening by Enzyme and Molecular Analyses in the New York City Minority Population. Genet Test Mol Biomark 2016;20(9):504-9.

13. van der Hout S, Holtkamp K, Henneman L, de Wert G, Dondorp W. Advantages of expanded universal carrier screening: what is at stake? Eur J Hum Genet. 2016;25(1):17-21.

14. Muncie HL, Campbell JS. Alpha and beta thalassemia. Am Fam Physician. 
2009;80(4):339-44.

15. Galanello R, Origa R. Beta-thalassemia. Orphanet J Rare Dis. 2010;5(1):1-15.

16. Cousens NE, Gaff CL, Metcalfe SA, Delatycki MB. Carrier screening for Beta-thalassaemia: a review of international practice. Eur J Hum Genet. 2010;18(10):1077-83.

17. Kalokairinou EM. The experience of $\beta$-thalassemia and its prevention in Cyprus. Med Law. 2007;26:291-307.

18. ACOG Committee. Practice bulletin No 78 . Haemoglobinopathies in pregnancies. Obstet Gynecol. 2007;109(1):229-37.

19. Cousens NE, Gaff CL, Metcalfe SA, Delatycki MB. Carrier screening for Beta-thalassaemia: a review of international practice. Eur J Hum Genet. 2010;18(10):1077-83.

20. Cao A, Galanello FR, Melis MA, Angius A, Ximenes A, Rosatelli A et al. Prevention of Homozygous /8-Thalassemia by Carrier Screening and Prenatal Diagnosis in Sardinia. Am J Hum Genet. 1981;33(4):592.

21. Cao A. Carrier screening and genetic counselling in $\beta$-thalassemia. Int J Hematol. 2002;76(2):10513.

22. Holtkamp KCA. Henneman L, Gillie JJP, Meijers-Heijboer H, Cornel MC, Lakeman P. Direct-to-consumer carrier screening for cystic fibrosis via a hospital website: a 6 year evaluation. J. Community Genet. 2018;10(2):249-57.

23. Castellani C, Macek Jr M, Cassiman JJ, Duff A, Massie J, ten KateLP et al. Benchmarks for Cystic Fibrosis carrier screening: A European consensus document. J Cyst Fibros. 2010;9(3):165-78.

24. Doksum T, Bernhardt BA, Holtzman NA.Carrier Screening for Cystic Fibrosis among Maryland Obstetricians before and after the $1997 \mathrm{NIH}$ Consensus Conference. 5(2):111-6.

25. Ioannou L, McClaren BJ, Massie J, Lewis S, Metcalfe SA, Forrest L et al. Population-based carrier screening for cystic fibrosis: a systematic review of 23 years of research. Genet. Med. 2014;16(3):207-16.

26. Rowe CA, Wright CF. Expanded universal carrier screening and its implementation within a publicly funded healthcare service. $\mathrm{J}$ Community Genet. 2020;11(1):21-38.

27. Wapner RJ.Acase for improved carrier screening. Contemporary ob/gyn. 2020;64(9):31-35.

28. Cecchi AC, Vengoechea ES, Kaseniit KE, Hardy MW, Kiger LA, Mehta N et al. Screening for TaySachs disease carriers by full-exon sequencing with novel variant interpretation outperforms enzyme testing in a pan-ethnic cohort. Mol Genet Genomic Med. 2019;7(8):e836.

29. Lazarin GA, Haque IS, Nazareth S, Iori K,
Patterson AS, Jacobson AL et al. An empirical estimate of carrier frequencies for $400+$ causal Mendelian variants: results from an ethnically diverse clinical sample of 23,453 individuals. Genet Med. 2013;15(3): 178-86.

30. Evolving Science of Genetic Carrier Screening. AJMC Perspectives. 2018

31. Grody WW, Thompson BH, Gregg AR, Bean LH, Monaghan KG, Schneider A et al. ACMG position statementon prenatal/preconception expanded carrier screening. Genet Med. 2013;15(6):482-3.

32. Srinivasan BS, Evans EA, Flannick J, Patterson AS, Chang CC, Pham T et al. Reproductive biomedicine online. 2010;21(4):537-51.

33. Azimi M, Schmaus K, Greger V, Neitzel D, Rochelle R, Dinh T. Carrier screening by nextgeneration sequencing: health benefits and cost effectiveness. Mol Genet Genomic Med. 2016;4(3):292-302.

34. Di Resta C, Galbiati S, Carrera P, Ferrari M. Next-generation sequencing approach for the diagnosis of human diseases: open challenges and new opportunities. 2018;29(1): 4-14.

35. Haque IS, Lazarin GA, Kang HP, Evans EA, Goldberg JD, Wapner JD. Modeled Fetal Risk of Genetic Diseases Identified by Expanded Carrier Screening. JAMA. 2016;316(7):734-42.

36. Holtkamp CA, Mathijssen IB, Lakeman P, van Maarle M, Dondorp WJ, Henneman L et al. Factors for successful implementationbased expanded carrier screening: learning from existing initiatives. Eur. J. Public Health. 2016;27(2):372-7.

37. Ben-Shachar R, Svenson A, Goldberg JD, Muzzey D. A data-driven evaluation of the size and content of expanded carrier screening panel. Genet Med. 2019;21(9):1931-9.

38. Lazarin GA, Hawthorne F, Collins NS, Platt EA, Evans EA, Haque IS. Systematic Classification of Disease Severity for Evaluation of Expanded Carrier Screening Panels. PLoS One, 2014;9(12):e114391.

39. Platinga M, Birnie E, Abbott KM, Sinke RJ, Lucassen AM, Schuurmans M et al. Populationbased preconception carrier screening: how potential users from the general population view a test for 50 serious diseases. Eur J Hum Genet. 2016;24(10):1417-23.

40. Bell CJ, Dinwiddle DL, Miller NA, Hateley SL, Ganusova EE, Mudge J et al. Carrier Testing for Severe Childhood Recessive Diseases by Next-Generation Sequencing. Sci Transl Med. 2011;3(65):65ra4-65ra4.

41. Beachuamp KA, Muzzey D, Wong KK, Hogan GJ, Karimi K, Candille SI et al. Systematic design and comparison of expanded carrier 
screening. Genet Med. 2018;20(1):55-63.

42. Wienke S, Brown K, Farmer M, Strange C. Expanded carrier screening panels-does bigger mean better? J Community Genet. 2014;5(2):191-8.

43. Dungan J. Expanded carrier screening: what the reproductive endocrinologist needs to know. Fertil Steril. 2019;109(2):183-9.

44. Prior TW. Next-generation carrier screening: are we ready? Genome Med. 2014;6(8):1-3.

45. Platinga M, Birnie E, Schuurmans J, Buitenhuis AH, Boersma E, Lucassen AM et al. Expanded carrier screening for autosomal recessive conditions in health care: Arguments for a couple-based approach and examination of couples' views. Prenat Diagn. 2018;39(5):36978.

46. Capalbo A, Valero RA, Jimenez-Almanaz J, Pardo PM, Fabiani M, Jimenez D et al. Optimizing clinical exome design and parallel gene-testing for recessive genetic conditions in preconception carrier screening: Translational research genomic data from 14,125 exomes. PLoS Genet. 2019;15(10):e1008409.

47. Rose NC, Wick M. Current recommendations: Screening for Mendelian disorders. Semin Perinatol. 2016;40(1):23-8.

48. Schuurmans J, Birnie E, van den Heuvel LM, Platinga M, Lucassen A, van der Kolk DM et al. Feasibility of couple-based carrier screening offered by general practitioners. Eur J Hum Genet. 2019;27(5):691-700.

49. Leung ML, McAdoo S, Watson D, Stumm K, Harr M, Wang Xiang et al. A transparent approach to calculate detection rate and residual risk for carrier screening. J Mol Diagn. 2021;23(1):91-102.

50. Lazarin GA, Haque IS. Expanded carrier screening: A review of early implementation and literature. Semin Perinatol 2016;40(1):2934.

51. Fridman H, Behar DM, Carmi S, Levy-Lahad E. Preconception carrier screening yield: effect of variants of unknown significance in partners of carriers with clinically significant variants. Genet Med. 2020;22(3): 646-53.

52. Xi Y, Chen G, Lei C, Wu J, Zhang S, Xiao $\mathrm{M}$ et al. Expanded carrier screening in Chinese patients seeking the help of assisted reproductive technology. Mol Genet Genomic Med. 2020;8:e1340.

53. Edwards JG, Feldman G, Goldberg J, Gregg AR, Norton ME, Rose NC et al. Expanded carrier screening in reproductive medicinepoints to consider: a joint statement of the American College of Medical Genetics and
Genomics, American College of Obstetricians and Gynecologists, National Society of Genetic Counselors, Perinatal Quality Foundation, and Society for Maternal-Fetal Medicine. Obstet Gynecol. 2015;125(3):653-62.

54. Abacan M, Alsubaie L, Borlow-Stewart $\mathrm{K}$, Caanen B, Cordier C, Courtney E et al. The Global State of the Genetic Counseling Profession. Eur J Hum Genet. 2019;27(2):18397.

55. Fakih A, Spector-Bagdady K. Should clinicians leave "expanded" carrier screening decisions to patients? AMA J Ethics. 2019;21(10):E858.

56. Cho D, McGowan ML, Metcalfe J, Sharp RR. Expanded carrier screening in reproductive healthcare: perspectives from genetics professionals. Hum. Reprod. 2013;28(6):172530 .

57. Kraft SA, Duenas D, Wilfond BS, Goddard KAB. The evolving landscape of expanded carrier screening: challenges and opportunities. Genet. Med. 2019;21(4):790-7. 